\title{
Hierarchical-information-based characterization of multiscale structured surfaces
}

\author{
Chi Fai Cheung (2) ${ }^{1 *}$, Mingyu Liu1, Richard Leach (2)2, Xiaobing Feng², Chenyang Zhao ${ }^{1}$ \\ ${ }^{1}$ Partner State Key Laboratory of Ultraprecision Machining Technology, Department of Industrial and Systems Engineering, The Hong Kong Polytechnic University, \\ Hung Hom, Kowloon, Hong Kong \\ ${ }^{2}$ Manufacturing Metrology Team, Faculty of Engineering, University of Nottingham, Nottingham, United Kingdom
}

This paper presents a hierarchical-information-based characterization method for multiscale structured surfaces. The method makes use of a priori information of the multiscale surface and a hierarchical segmentation-registration (HSR) algorithm to firstly divide the measurement dataset into segments, which are then registered with the designed geometry of the microstructure. The registered information is used to reconstruct the large scale topography. Multiple scales of surface information are decomposed hierarchically by the HSR algorithm. Hence, the errors in different scales are determined. Simulation and experimental results show that the hierarchical-information-based characterization method is accurate and effective in the characterization of multiscale structured surfaces.

Information, Surface, Characterization

\section{Introduction}

Multiscale structured surfaces (MSSs), such as microlens arrays [1], micro-pyramids [2] and sinusoidal micro-structures [3], are playing an important role in advanced optics [4] and bio-inspired applications [5]. Small-scale structured patterns are embedded in large-scale flat or curved substrates using multiscale surface design processes, where the different scales of geometry have different functions. Whilst methods for machining of MSSs have been explored [1, 2], only qualitative methods for their characterization have been applied [2], or the surface is characterized without separating out the different scale geometries [1].

Traditional top-down methods for MSS characterization based on wavelength decomposition, such as Gaussian filtering [6] and wavelet analysis [7], can separate the multiscale topography to obtain the large-scale substrate and the small-scale microstructure by setting appropriate cut-off wavelengths. However, such cut-off wavelengths are difficult to determine, and the filtering/separation can result in significant surface distortion due to the transmission characteristics of the filters [8]. This distortion issue is especially critical for MSS since the microstructures can be as small as several micrometres, which places limits on the available measurement technologies. Several methods $[9,10]$ have been developed for characterization of MSSs but the accuracy is still a question.

This paper proposes a hierarchical-information-based method for characterization of MSSs. The method is based on a hierarchical segmentation-registration algorithm. Simulations and experiments are conducted to demonstrate the capability and effectiveness of the method.

\section{Hierarchical-information-based characterization method}

As opposed to the traditional top-down methods, the proposed hierarchical-information-based characterization method is a bottom-up method which is based on a hierarchical segmentation-registration (HSR) algorithm. Figure 1 shows a schematic diagram of the HSR algorithm. The method firstly divides the MSS into segments, where each segment is one fundamental geometrical element (tessellation) of the topography. Secondly, the segments are registered to the designed microstructure to determine the topographical errors and the locations of the elements are determined. Lastly, the substrate is reconstructed by using the locations of the elements and the design information of the MSS. Hence, the form error in the substrate can be determined.

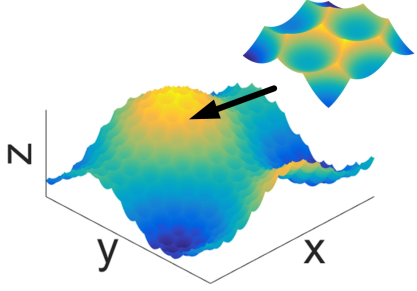

(a) Multiscale structured surface

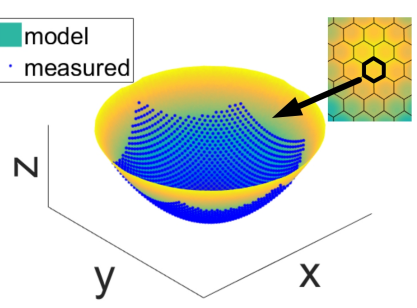

(c) Registration of microstructures

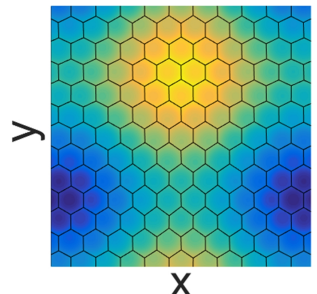

(b) Segmentation

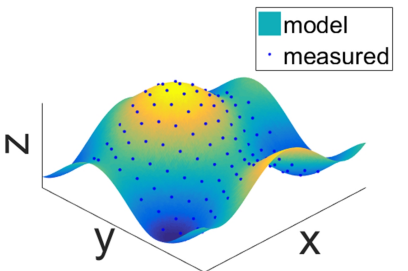

(d) Registration of substrate
Figure 1. Schematic overview of the HSR algorithm.

\subsection{Segmentation of microstructures}

A key challenge of the proposed HSR algorithm is the segmentation of the microstructures. The intrinsic features, such as the Gaussian curvature (GC) and mean curvature (MC) [11], are used for pattern analysis to identify the microsctructure segments. Taking a lens array, a sinusoidal array and a pyramid array as examples, the associated Gaussian curvature or mean curvature are determined, as shown in Figure 2. By setting a threshold, data points representing features, such as corners and 
edges of specific microstructures, can be identified and then clustered by distance. Hence, the microstructures can be further identified using a priori information about the design. Using the curvature information is essential, especially when the microstructures are designed on a curved substrate. Since the outliers in the measured topography significantly affect the calculation of Gaussian curvature and mean curvature, a statistical outlier removal filter [12] is used to remove them.

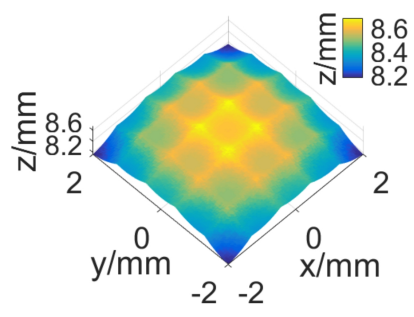

(a) Lens array

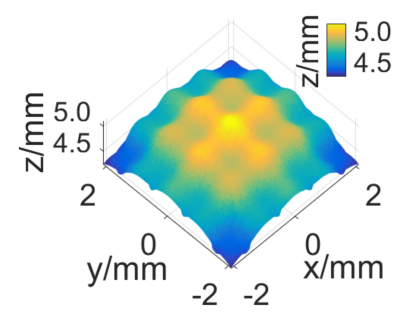

(c) Sinusoidal array
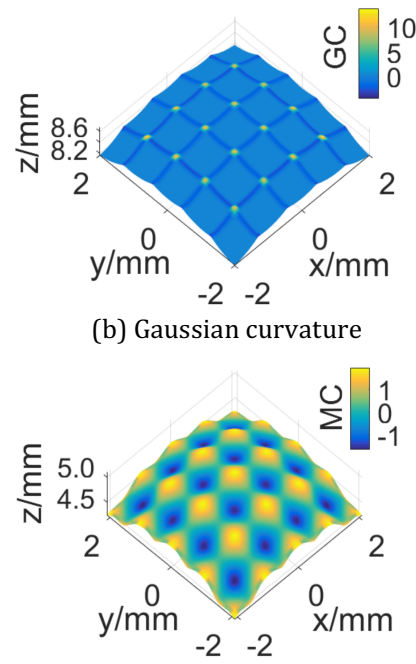

(d) Mean curvature (b) Gaussian curvature

Figure 2. Curvatures of different structured surfaces.

\subsection{Registration of microstructures and determination of key} points

After the MSS is divided into segments, topographical error in each microstructure can be determined by registering with the designed microstructure. This is done by the iterative closest point (ICP) algorithm [13]. After registration, each segment has an associated transformation matrix. Using the priori information of the MSS design, a key point on the measured microstructure is associated with the nominal geometry of the designed microstructure in order to designate the location of the microstructure. For example, the centre point is the key point of the spherical microlens. The coordinates of the key point $K_{t}$ of the segment is then determined by:

$K_{t}=\operatorname{Tr} \times K$

where $K$ is the coordinate of the key point for the designed microstructure, and $T r$ is the reversed transformation matrix associated with the registration, which rotates and translates in the reversed directions.

\subsection{Reconstruction and registration of the substrate}

Reconstruction of the substrate is performed by making use of both the registration results for the microstructures and the a priori information about the MSS (i.e. substrate design, microstructure geometry and machining method). Without loss of generality, the principle is illustrated in Figure 3 with the same MSS as shown in Figure 1. Assume the MSS has the following design characteritics:

1. A large-scale substrate.

2. The substrate is offset by a distance which is used for locating the centres of the microlenses.

3. The microlenses are superimposed on to the substrate with the designed shape (i.e. a sphere with radius $R$ ).

In the reconstruction and registration process, the procedure is essentially the reverse of the above design steps, i.e.:
1. The centres of microlenses are determined by registration of the microstructures.

2. The offset of the substrate is reconstructed by using the centre points of the microlenses.

3. The substrate is offset in the reversed direction according to the a priori information.

4. The reconstructed substrate is registered to the design substrate to determine the form error.

The offset substrate in step 2 is reconstructed by interpolating the data points using the Gaussian process [14], which is essential to allow form correction in the machining process.

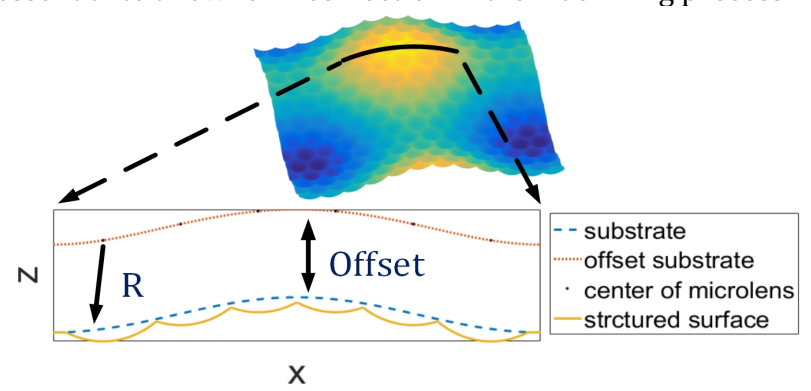

Figure 3. Design information of the example MSS.

\section{Method verification}

\subsection{Accuracy analysis with simulation}

To evaluate the accuracy of the proposed method, a designed surface is simulated with a microlens array on an f-theta surface, as shown in Figure 4. The $\mathrm{f}$-theta surface is determined by:

$z=-x^{2} / 250+x^{4} / 92000-y^{2} / 25$

where $x \in[-20,20] \mathrm{mm}$ and $y \in[-7.5,7.5] \mathrm{mm}$. Individual microlenses are spherical with $0.8 \mathrm{~mm}$ radius of curvature, and the sphere centres are located on the surface with $0.76 \mathrm{~mm}$ offset to the substrate. The distance between the microlenses and sampling resolution in both the $x$ and $y$ directions is $0.35 \mathrm{~mm}$ and $0.01 \mathrm{~mm}$, respectively. The result of the segmentation for the surface is shown in Figure 5 (different colours represent different segments). The error maps on the scale of the microlenses and on the scale of the f-theta substrate were determined and the results are shown in Figure 6.

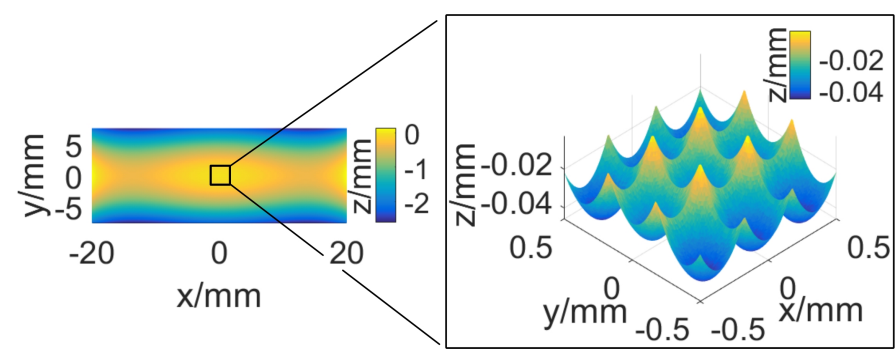

Figure 4. Microlens array on an f-theta surface.

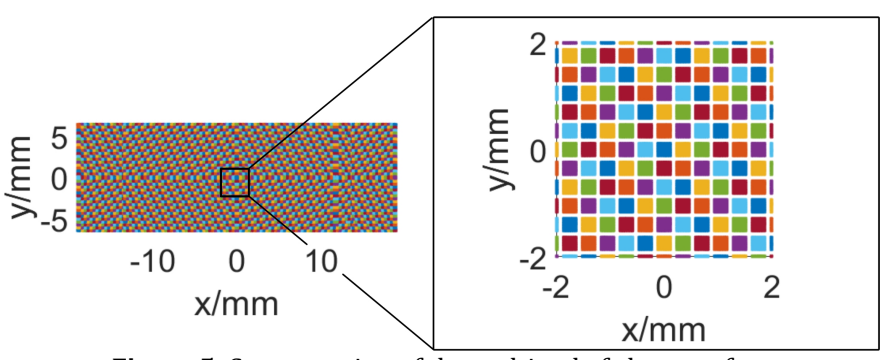

Figure 5. Segmentation of the multiscale f-theta surface.

The simulated MSS is an ideal surface without any errors. Hence, the determined errors are attributed by the proposed 
method including surface registration, surface reconstruction, etc. Both results show the errors are small. The peak-to-valley (PV) value for the microlens and substrate are approximately $0.05 \mu \mathrm{m}$ and $0.08 \mu \mathrm{m}$, respectively. The root-mean-square (RMS) value of the error maps for the microlenses and the substrate are 0.0280 $\mu \mathrm{m}$ and $0.0089 \mu \mathrm{m}$, respectively. The results demonstrate the proposed method is accurate as summarised in Table 1.

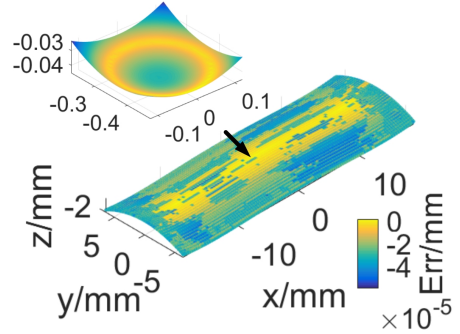

(a) Error map for microstructures

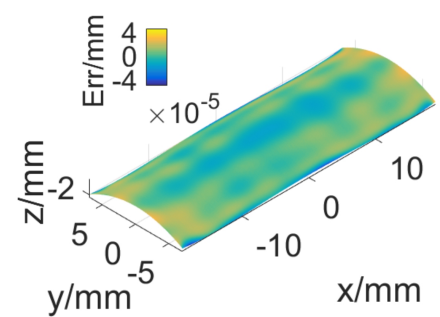

(b) Error map for the substrate
Figure 6. Error maps for microstructure and the substrate of the multiscale f-theta surface.

Table 1

Errors for microstructure and substrate of the multiscale f-theta surface

\begin{tabular}{lll}
\hline & Microstructure & Substrate \\
\hline Peak-to-valley value $(\mu \mathrm{m})$ & 0.05 & 0.08 \\
Root-mean-square value $(\mu \mathrm{m})$ & 0.0280 & 0.0089 \\
\hline
\end{tabular}

\subsection{Measurement results}

Two different kinds of MSSs were produced by multi-axis diamond machining with a slow tool servo (STS) method. One is a microlens array machined on to a sinusoidal surface, which was produced using a Moore Nanotech 350FG ultra-precision machine. The sinusoidal surface is designed with the following:

$$
z=0.02[\sin (0.2 \pi x)+\cos (0.2 \pi y)]
$$

where $x, y \in[-10,10] \mathrm{mm}$ and $\sqrt{x^{2}+y^{2}}<10 \mathrm{~mm}$. The microlens is designed as a spherical surface with a $4 \mathrm{~mm}$ radius of curvature and the sphere centre is located $3.95 \mathrm{~mm}$ above the substrate. As a result, the height of every microlens is $50 \mu \mathrm{m}$. The centres of the microlenses are distributed on an equilateral triangle with $2 \mathrm{~mm}$ side length, hence the microlenses form a hexagonal shape in the lateral direction. The MSS was measured by a coherence scanning interferometer (CSI) with a $20 \times$ objective and using the stitching function. The machined workpiece and the measured topography are shown in Figure 7.

With the proposed HRS method, the MSS was successfully characterized at different scales, as shown in Figure 8. Figure 8(a) shows the segmentation result and Figure 8(b) shows the error map for the microlens array, where the PV value is approximately $2 \mu \mathrm{m}$ over the whole surface and the RMS value is $0.25 \mu \mathrm{m}$. The error map for a particular microlens is also presented in Figure 8(c), where a wave pattern which may be caused by the motion error of the machine tool is evident. Figure 8(d) shows the error map for the substrate, where the PV value is $7.9 \mu \mathrm{m}$ and the RMS value is $1.8 \mu \mathrm{m}$ (see Table 2). As the local gradient and the surface roughness of the diamond-turned workpiece are both small, the measurement uncertainty of the CSI is in nanometre level [15], which has little influence on the result and can be neglected in the calculation. The relatively large error in the substrate is due to the relatively large cutting forces in STS machining of the structured surface, since the cutting speed is much slower than when machining continuous freeform surfaces. The error map for the substrate shows a funnel-shaped pattern, where the outer area has a larger error than the inner area. The error of the substrate may be caused by a tilting error of the spindle, which is affected by the cutting force. Basically, the error caused by tilting is larger in the outside area than in the centre when assuming a constant cutting force. A thorough study will be conducted to investigate this issue in the future work. The results show that the separation of the errors for different scales significantly enhances the understanding of the machining process. It also provides a powerful method to further improve the accuracy of MSS potentially by error compensation and this will be investigated in the future work.

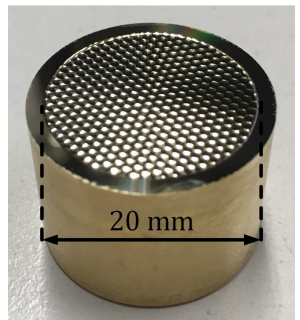

(a) Measurement setup

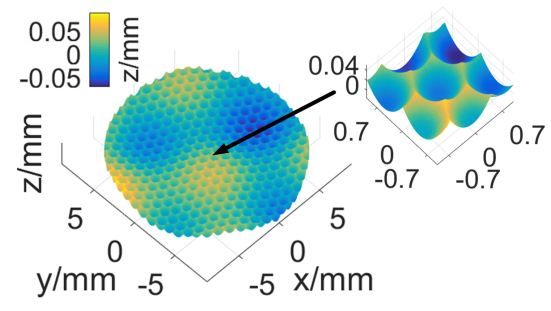

(b) Measured topography
Figure 7. Measurement of the diamond turned microlens array on a sinusoidal surface.

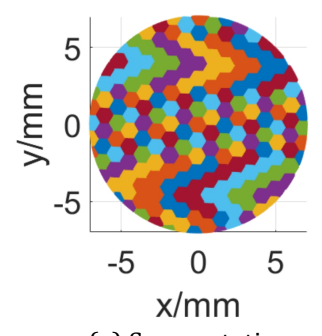

(a) Segmentation

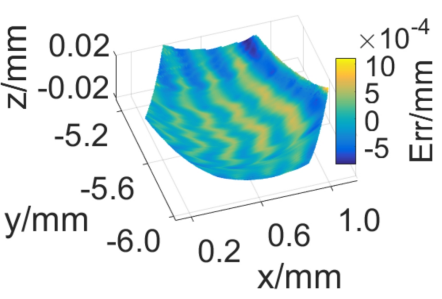

(c) Error map for a single microlens

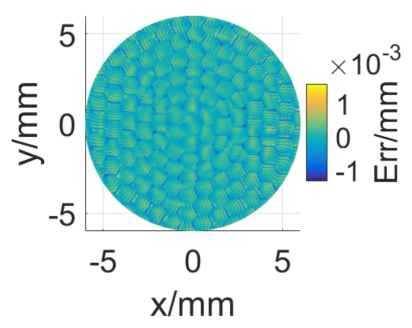

(b) Error map for microlens

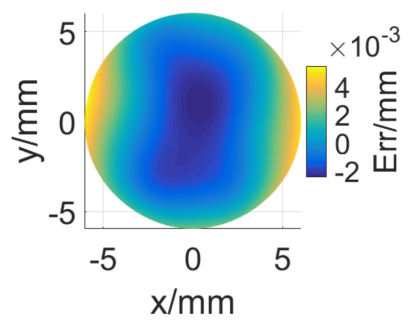

(d) Error map for substrate
Figure 8. Characterization results of the microlens array on a sinusoidal surface.

Table 2

Errors for microstructure and substrate of the microlens array on a sinusoidal surface

\begin{tabular}{lll}
\hline & Microstructure & Substrate \\
\hline Peak-to-valley value $(\mu \mathrm{m})$ & 2.0 & 7.9 \\
Root-mean-square value $(\mu \mathrm{m})$ & 0.25 & 1.80 \\
\hline
\end{tabular}

A further MSS design was a sinusoidal array on a roller surface. The radius of the cylinder is $3 \mathrm{~mm}$ and the height is $6.28 \mathrm{~mm}(2 \pi)$. The sinusoidal structure is designed in a cylindrical coordinate system and the radial distance (RD) is determined by: $r=3+0.02[\sin (5 z)+\cos (20 \theta)-2]$

where $z \in[0,6.28] \mathrm{mm}$ is the height along the cylindrical axis, and $\theta \in\left[0,360^{\circ}\right)$ is the azimuth. The MSS was machined by a Moore Nanotech 450FG ultra-precision machine with a STS and measured using a point autofocus instrument (PAI) with a $100 \times$ objective. The sampling resolution in the axis and circumferential 
directions are both approximately $50 \mu \mathrm{m}$. The measurement setup and the results are shown in Figure 9.

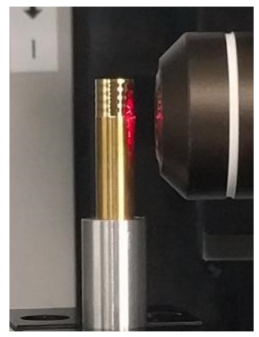

(a) Measurement setup

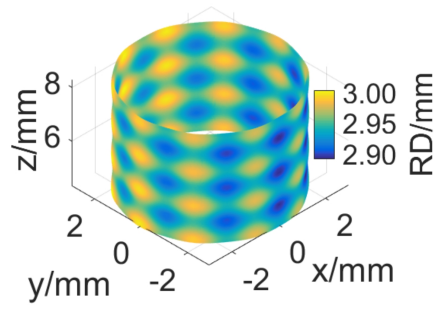

(b) Measurement result
Figure 9. Measurement of the diamond turned sinusoidal array on a roller surface.

The characterization result of the MSS is shown in Figure 10. Figure 10(a) shows the mean curvature of the MSS, where the peaks and valleys are clearly determined. The MSS is segmented by using the curvature information and the result is shown in Figure $10(\mathrm{~b})$. The topographical error in the sinusoidal structure is determined as shown in Figure $10(\mathrm{c})$, where the PV value is $10.7 \mu \mathrm{m}$ and the RMS value is $0.37 \mu \mathrm{m}$. The large PV value is caused by tool marks, which are left in the rough machining of the workpiece. The topographical error in the cylindrical substrate is shown in Figure 10(d), where the PV value is $1.7 \mu \mathrm{m}$ and the RMS value is $0.39 \mu \mathrm{m}$. The measurement uncertainty of the PAI is determined to be in nanometre level [16] and thus it has little influence on the result and can be neglected in the experiment. The topographical error in the substrate shows that the radius of the cylinder is smaller in the top area; this is most probably caused by a tilting error of the spindle of the diamond turning machine. The results are summarised in Table 3.

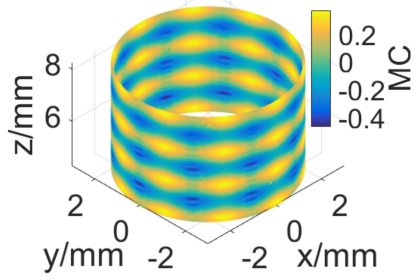

(a) Mean curvature

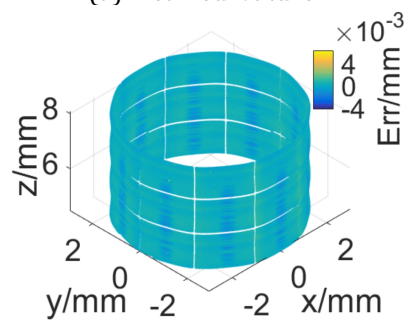

(c) Error map for microstructures
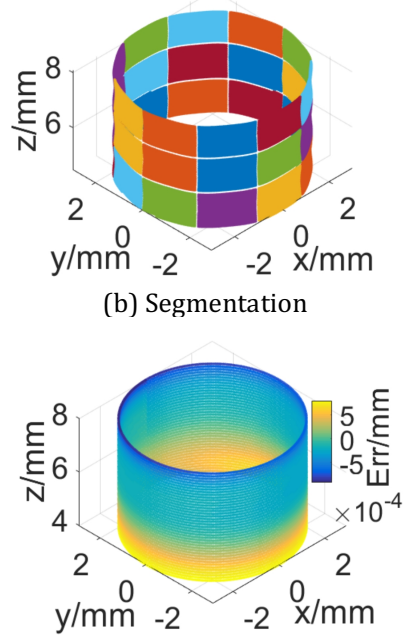

(d) Error map for the substrate (b) Segmentation

Figure 10. Characterization results of the sinusoidal array on a roller surface.

Table 3

Errors for microstructure and substrate of the sinusoidal array on a roller surface

\begin{tabular}{lll}
\hline & Microstructure & Substrate \\
\hline Peak-to-valley value $(\mu \mathrm{m})$ & 10.7 & 1.7 \\
Root-mean-square value $(\mu \mathrm{m})$ & 0.37 & 0.39 \\
\hline
\end{tabular}

Both experiments demonstrate the effectiveness of the proposed hierarchical-information-based characterization method. Moreover, the advantages and uniqueness of the method are shown, i.e. the topographical errors at different scales can be precisely determined. The information about the topographical error can be further utilised for functional testing according to the design of the MSS. Moreover, error compensation is possible with the help of the error information obtained. Functional tests to determine the characteristic of the MSSs will be investigated in the future work.

\section{Conclusion}

This paper presents a hierarchical-information-based characterization method for multiscale structured surfaces (MSSs). The method is based on the use of a priori design data and manufacturing information about the MSS and a hierarchical segmentation-registration algorithm. The surface is first divided into segments according to the geometry of the designed microstructure and the topographical errors of the microstructures are determined by registration. The substrate is then reconstructed using the registration result and hence its form error can be determined. Simulation results show that the proposed method is accurate at the sub-micrometre level. Experimental results show that the proposed method successfully characterizes MSSs and determines errors at different scales.

\section{Acknowledgements}

The work described in this paper was mainly supported by a grant from the Research Grants Council of the Government of the Hong Kong Special Administrative Region, China (Project No. 15202717). The authors also sincerely thank the EMPIR organization. The EMPIR is jointly funded by the EMPIR participating countries within EURAMET and the European Union (15SIB01: FreeFORM).

\section{References}

[1] Li, L., Yi, A.Y., 2009, Microfabrication on a curved surface using 3D microlens array projection, Journal of Micromechanics and Microengineering, 19/10:105010.

[2] To, S., Zhu, Z.W., Zeng, W.H., 2015, Novel end-fly-cutting-servo system for deterministic generation of hierarchical micro-nanostructures, CIRP Annals Manufacturing Technology, 64/1:133-136.

[3] Gao, W., Tano, M., Sato, S., Kiyono, S., 2006, On-machine measurement of a cylindrical surface with sinusoidal micro-structures by an optical slope sensor, Precision engineering, 30/3:274-279.

[4] Fang, F.Z., Zhang, X.D., Weckenmann, A., Zhang, G.X., Evans, C., 2013, Manufacturing and measurement of freeform optics, CIRP Annals - Manufacturing Technology, 62/2:823-846.

[5] Malshe, A., Rajurkar, K., Samant, A., Hansen, H.N., Bapat, S., Jiang, W.P., 2013, Bioinspired functional surfaces for advanced applications, CIRP Annals - Manufacturing Technology, 62/2:607-628.

[6] Jiang, X., 2010, Robust solution for the evaluation of stratified functional surfaces, CIRP Annals - Manufacturing Technology, 59/1:573-576.

[7] Raja, J., Muralikrishnan, B., Fu, S., 2002, Recent advances in separation of roughness, waviness and form, Precision Engineering, 26/2:222-235.

[8] Whitehouse, D.J., 2010, Handbook of surface and nanometrology, CRC press.

[9] Whitehouse, D.J., 2016, Undefined freeform surfaces having deterministic structure: issues of their characterization for functionality and manufacture, Surface Topography: Metrology and Properties, 4/3:035001.

[10] Jiang, X., Abdul-Rahman, H.S., Scott, P.J., 2013, Multi-scale freeform surface texture filtering using a mesh relaxation scheme, Measurement Science and Technology, 24/11:115001.

[11] Cheung, C.F., Kong, L.B., Ren, M.J., Whitehouse, D., To, S., 2012, Generalized form characterization of ultra-precision freeform surfaces, CIRP Annals - Manufacturing Technology, 61/1:527-530.

[12] Rusu, R.B., Cousins, S., 2011, 3D is here: Point Cloud Library (PCL), 2011 IEEE International Conference on Robotics and Automation, 9-13 May 2011.

[13] Besl, P.J., McKay, N.D., 1992, A method for registration of 3-D shapes, IEEE Transactions on pattern analysis and machine intelligence, 14/2:239-256.

[14] Liu, M.Y., Cheung, C.F., Cheng, C.H., Su, R., Leach, R.K., 2017, A Gaussian process and image registration based stitching method for high dynamic range measurement of precision surfaces, Precision Engineering 50/99-106.

[15] Liu, M.Y., Cheung, C.F., Ren, M.J., Cheng, C.H., 2015, Estimation of measurement uncertainty caused by surface gradient for a white light interferometer, Applied Optics, 54/29:8670-8677. 
[16] Maculotti, G., Feng, X., Galetto, M., Leach, R.K., 2018, Noise evaluation of a point autofocus surface topography measuring instrument, Measurement Science and Technology, In press 